\section{Loss of maternal Trim28 causes male-predominant early embryonic lethality}

\author{
Abhishek Sampath Kumar, ${ }^{1}$ Michelle K.Y. Seah, ${ }^{1,6}$ \\ Ka Yi Ling, ${ }^{1,6}$ Yaju Wang, ${ }^{1}$ Joel H.L. Tan, ${ }^{1,2}$ \\ Sandra Nitsch, ${ }^{1}$ Shu Ly Lim, ${ }^{1}$ \\ Chanchao Lorthongpanich, ${ }^{3}$ Heike Wollmann, ${ }^{4}$ \\ Diana H.P. Low, ${ }^{5}$ Ernesto Guccione, ${ }^{2,5}$ \\ and Daniel M. Messerschmidt ${ }^{1,2}$
}

\begin{abstract}
${ }^{1}$ Developmental Epigenetics and Disease Group, Institute of Molecular and Cell Biology (IMCB), Agency for Science, Technology, and Research (A*STAR), Singapore 138673; ${ }^{2}$ Department of Biochemistry, Yong Loo Lin School of Medicine, National University of Singapore, Singapore $119074{ }^{3}$ Siriraj Center of Excellence for Stem Cell Research, Mahidol University, Bangkok 10700, Thailand; ${ }^{4}$ Next-Generation Sequencing Unit of DNA Sequencing Facility, IMCB, A*STAR, 138673, Singapore; ${ }^{5}$ Methyltransferases in Development and Disease Group, IMCB, A*STAR, Singapore 138673
\end{abstract}

Global DNA demethylation is a hallmark of embryonic epigenetic reprogramming. However, embryos engage noncanonical DNA methylation maintenance mechanisms to ensure inheritance of exceptional epigenetic germline features to the soma. Besides the paradigmatic genomic imprints, these exceptions remain ill-defined, and the mechanisms ensuring demethylation resistance in the light of global reprogramming remain poorly understood. Here we show that the Y-linked gene Rbmy1a1 is highly methylated in mature sperm and resists DNA demethylation post-fertilization. Aberrant hypomethylation of the Rbmy1a1 promoter results in its ectopic activation, causing male-specific peri-implantation lethality. Rbmy1a1 is a novel target of the TRIM28 complex, which is required to protect its repressive epigenetic state during embryonic epigenetic reprogramming.

Supplemental material is available for this article.

Received October 4, 2016; revised version accepted December 27, 2016.

Germ cells are highly differentiated cells that give rise to the next generation's embryo upon fertilization. Their unique epigenome, which reflects their specialization, must be reprogrammed for proper development. A hallmark of this process is genome-wide DNA demethylation, culminating in the open chromatin state of the epiblast (Messerschmidt et al. 2014). However, specialized genomic regions must retain DNA methylation for inheritance

[Keywords: DNA methylation; epigenetics; Rbmy; reprogramming; splicing; Trim28]

${ }^{6}$ These authors contributed equally to this work. Corresponding author: danielm@imcb.a-star.edu.sg

Article published online ahead of print. Article and publication date are online at http://www.genesdev.org/cgi/doi/10.1101/gad.291195.116. Freely available online through the Genes \& Development Open Access option. of vital epigenetic germline features to the soma. Prominent among them are genomic imprints, which engage noncanonical DNA methylation maintenance mechanisms to become reprogramming-resistant. Despite the importance of this process, only a few reprogramming-resistant regions and their functions have been described beyond genomic imprints, and the mechanisms allowing methylation maintenance in light of epigenetic reprogramming are poorly understood (Branco et al. 2016).

In early embryos, maintenance DNA methyltransferase (DNMT1) levels are low to facilitate global demethylation, yet methylation is still maintained by DNMT1 at imprinted regions (Hirasawa et al. 2008). Our previous work revealed a fundamental role of maternal TRIM28 in this process, achieved through TRIM28's recruitment by the Krueppel-associated box domain zinc finger protein (KRAB-ZFP) ZFP57 and noncanonical targeting of DNMT1 (Messerschmidt et al. 2012; Lorthongpanich et al. 2013).

Paralleling the imprinting defects in maternal Trim28 mutant embryos, we now expose a sex-specific early embryonic lethality phenotype. Our new findings show that, besides imprints, TRIM28 safeguards germline-tosoma inheritance of epigenetic features at other genomic regions in an exquisitely stage-dependent manner.

\section{Results and Discussion}

TRIM28 is essential for development and maternal or zygotic deletion (Supplemental Fig. S1A) and is embryonic-lethal (Cammas et al. 2000; Messerschmidt et al. 2012). In zygotic mutants, maternally inherited Trim28 gene products remain unperturbed, and embryos arrest at gastrulation. Removal of maternal Trim28 also results in embryonic lethality; however, timing and causality are remarkably variable, presumably owing to the mosaic nature of DNA methylation defects leading to variable gene expression (Messerschmidt et al. 2012; Lorthongpanich et al. 2013).

Despite the stochastic nature of the phenotype, we found $57 \%(n=252$ out of 444$)$ of maternal-null Trim28 (Trim28 $8^{\text {mat } \Delta /+}$ ) embryos to be resorbed immediately after implantation (Fig. 1A,B; Supplemental Fig. S1B; Messerschmidt et al. 2012). This means that Trim28 $8^{\text {mat } \Delta /+}$ blastocysts form functional trophectoderm (TE) and induce decidualization, yet merely half are capable of further development. In comparison, only $5 \%(n=7$ out of 142) of control (Trim28 $8^{f /+}$ ) embryos were found resorbed (Fig. 1A).

Preimplantation Trim28 $8^{\text {mat } \Delta /+}$ embryos are unperturbed (Messerschmidt et al. 2012). We therefore examined implanting embryonic day 4.5 (E4.5) embryos, finding variable degrees of morphological abnormalities among littermates. Abnormal embryos displaying fragmented, pyknotic nuclei coinciding with active Caspase 3 staining were found next to normal, expanded blastocysts (Fig. 1C). However, despite being morphologically abnormal, these embryos still showed normal lineage

(C) 2017 Kumar et al. This article, published in Genes \& Development, is available under a Creative Commons License (Attribution 4.0 International), as described at http://creativecommons.org/licenses/by/4.0/. 


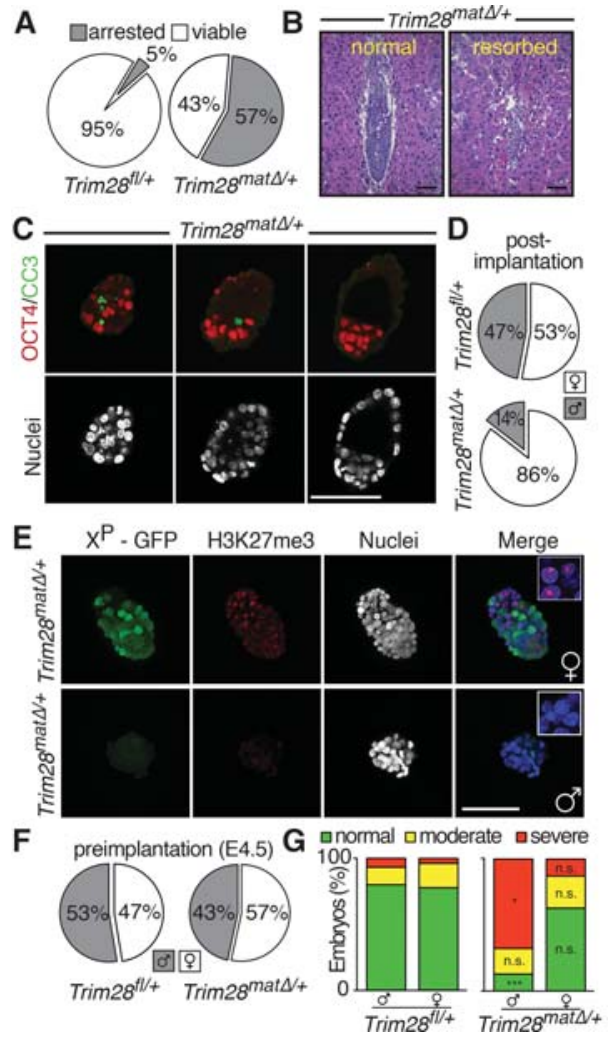

Figure 1. The absence of maternal Trim28 causes male-predominant early embryonic lethality. (A) Percentage of post-implantation embryonic lethality in Trim $28^{\mathrm{f} /+}$ and Trim $28^{\text {mat } \Delta /+}$ embryos. (B) H\&E-stained sections of deciduomas of normal and resorbed Trim2 $8^{\text {mat } / /+}$ littermates at embryonic day 6.5 (E6.5). (C) OCT3/4 and cleaved Caspase 3 (CC3) immunofluorescence staining of E4.5 Trim28 $8^{\text {mat } \Delta /+}$ littermates. $(D)$ Observed sex ratio in post-implantation mutant and control litters. (E) Female (top) and male (bottom) E4.5 Trim28 $8^{\text {mat } \Delta /+}$ embryos showing normal and abnormal morphology, respectively. $(F)$ Observed sex ratio in preimplantation mutant and control litters. $(G)$ Quantification of the morphological defects observed in mutant/control male/female embryos. Bars, $100 \mu \mathrm{m}$.

segregation, displaying inner cell mass (ICM) and TE markers (Fig. 1C; Supplemental Fig. S1C).

The frequency of peri-implantation lethality suggests a close to 1:1 segregation ratio of the phenotype despite embryos being genetically identical (Supplemental Fig. S1A). Furthermore, the Trim $28^{f / f}$ line was extensively backcrossed to the C57BL/6J genetic background, excluding a segregation of strain-specific determinants (Cammas et al. 2000; Messerschmidt et al. 2012). Instead, sex determination of embryos surviving beyond implantation revealed a remarkable sex ratio bias, with $86 \%(n=65$ out of 76) of the surviving Trim $28^{\text {mat } \Delta /+}$ embryos being female, suggesting a gonosome-linked phenotype. Control litters segregated as expected in a close to $1: 1$ ratio $(53 \%$ $[n=27]$ females and $47 \%[n=24]$ males) (Fig. 1D).

For faithful, noninvasive sexing of embryos, we used an X-linked GFP reporter (Supplemental Fig. S2; Hadjantonakis et al. 1998). We excluded Trim28 mutant-related loss of GFP expression in females by examining embryos for the presence or absence of typical punctate H3K27me3 staining labeling the inactivated $\mathrm{X}$ chromosome and conducted Sry genotyping and/or Xist expression analysis (Fig. 1E; data not shown). While females always showed reliable
GFP expression, male embryos remained GFP-negative, and $\mathrm{X}$ inactivation was not evident at E4.5. In contrast to post-implantation stages, the sex ratio remained balanced at E4.5, with $47 \% / 53 \%(n=57)$ females and males in control and $57 \% / 43 \%(n=156)$ females and males in mutant litters, respectively (Fig. 1F). However, when categorized morphologically (Supplemental Fig. S1D), a significant increase of severely defective mutant males was observed, while mutant females showed no significant changes in morphological categorization (Fig. 1G). Thus, the absence of maternal TRIM28 causes male-predominant peri-implantation embryonic lethality.

Sex-specific differences in mouse preimplantation embryos are limited to gonosomes, including X-chromosome dosage compensation in females. We found no indication of the characteristic H3K27me3 labeling of condensed X chromosomes in mutant males or a second condensed X chromosome in female cells, eliminating aberrant "imprinted" maternal X inactivation, possibly caused by exposure of the maternal $\mathrm{X}$ chromosome to the Trim28-null environment in the oocyte (Fig. 1E, insets; Supplemental Fig. S2B,C). Post-implantation Trim $28^{\text {mat } \Delta /+} / \mathrm{XX}^{\mathrm{P}}$-GFP females displayed GFP-negative and GFP-positive cells at comparable ratios (Supplemental Fig. S2), excluding the specific loss of cells relying on the maternally inherited, potentially "defective" X chromosome (50\% of cells in females and all cells in males).

Unable to find global X-linked defects, we analyzed gene expression in mutant $(n=12)$ and control $(n=7)$ blastocysts (Supplemental Table 1). Sixty-seven and 68 transcripts were up-regulated and down-regulated, respectively, clustering in 16 gene ontology categories (Supplemental Tables 2-3). X-linked genes were not enriched; five transcripts were moderately down-regulated, and one transcript was weakly induced. In contrast, one Y-linked transcript (Gm10352 or Rbmy1a1) was highly induced in mutants and virtually absent in controls. Exposure of the paternal genome to the Trim28-null environment after fertilization causes DNA demethylation of paternal imprints (Messerschmidt et al. 2012; Lorthongpanich et al. 2013). The absence of maternal TRIM28 may also relieve gene repression through hypomethylation. Focusing on the Y chromosome, we found that, other than Rbmy1a1, which showed a highly significant activation in mutants, other Y-linked genes are not expressed at all or not differentially expressed in mutants and controls (Fig. 2A). Rbmy1a1 is a multicopy gene ( 30 copies) (Soh et al. 2014) encoding a testis-specific RNA-binding protein involved in alternative mRNA splicing (Zeng et al. 2008). Nine copies are reliably annotated (GRCm38/ $\mathrm{mm} 10$ ) and encode for the full Rbmy1a1 ORF. For subsequent analyses, we therefore selected promoter and coding regions that are conserved among all annotated copies. Quantitative RT-PCR (qRT-PCR) analysis throughout preimplantation confirmed the dramatic Rbmy1a1 activation in individual Trim28 $8^{\text {mat } \Delta /+}$ embryos (Fig. 2B), occurring as early as the two-cell stage up to the late blastocyst. Neither control males nor any females showed Rbmy1a1 expression (Fig. 2B). Consistently, RBMY1A1 protein was detectable in mutant but not control males and was never detectable in females (Fig. 2C; Supplemental Fig. S3). In line with previously described mosaic loss of imprinting (Messerschmidt et al. 2012; Lorthongpanich et al. 2013), Rbmy1a1 expression was variable among Trim $28^{\text {mat } \Delta /+}$ male embryos, possibly explaining the survival of a few individuals beyond early 

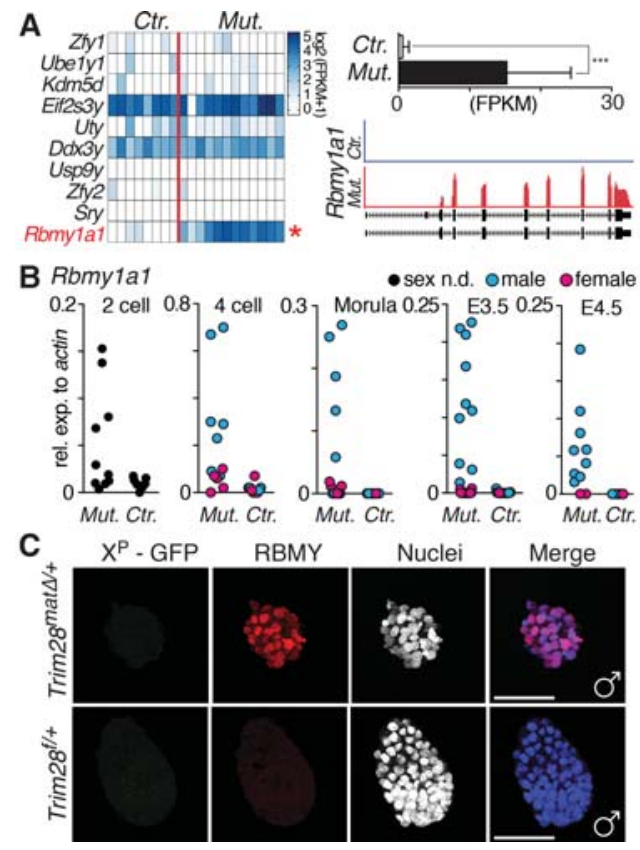

Figure 2. Male-specific transcriptional changes in Trim $28^{\text {mat } /++}$ embryos. (A) RNA sequencing (RNA-seq) of individual mutant and control E3.5 blastocysts. The heat map represents $\log _{2}(\mathrm{FPKM}+1)$ values of Y-chromosome-encoded genes. $(B)$ qRT-PCR analysis of Rbmy1a1 expression in preimplantation embryos from the two-cell to the late blastocyst stage in mutants and controls. Sex was not determined by Xist or X-GFP expression (not possible at the two-cell stage). (C) Immunofluorescence staining for RBMY1A1 in mutant and control E4.5 male embryos. Bar, $100 \mu \mathrm{m}$.

implantation stages (Fig. 1D). In summary, loss of maternal TRIM28 results in the ectopic activation of the paternally inherited gene Rbmyla1 as early as the two-cell stage.

TRIM28 mediates de novo methylation and DNA methylation maintenance (Wiznerowicz et al. 2007; Quenneville et al. 2011; Messerschmidt et al. 2012; Rowe et al. 2013). We thus examined a conserved Rbmyla1 promoter region containing eight CpGs in sperm, embryos, embryonic stem cells (ESCs), and somatic cells (Fig 3; Supplemental Fig S4). In sperm the Rbmy1a1 promoter is highly methylated $(88 \%)$. Surprisingly, this hypermethylation is maintained in wild-type blastocysts (average $80 \% ; n=3$ litters), suggesting resistance to epigenetic reprogramming post-fertilization comparable with genomic imprints. In contrast, the Oct3/4 promoter serving as a control was fully demethylated at the blastocyst stage (Supplemental Fig. S4). However, in Trim2 $8^{\text {mat } /++}$ blastocysts, the reprogramming resistance was lost, and dramatic hypomethylation of the Rbmyla1 promoter ensued; only $21 \%$ of CpGs were methylated in mutant blastocysts (Fig. 3A; Supplemental Fig. S4E). This is a significant reduction compared with controls $(P=0.0042)$ (Fig. 3A) and is consistent with Rbmyla1 activation in Trim $28^{\text {mat } \Delta /+}$ male embryos. Hypomethylation was not found in hepatocyte-specific Trim28 knockout livers (Fig. 3A) or, remarkably, Trim28 shRNA knockdown ESCs (Supplemental Fig. S4F,G), suggesting an early embryo-specific role of TRIM28 in Rbmy1a1 repression.

A study found that TRIM28-mediated methylation of foreign DNA is established mainly between E0.5 and
E2.5 and subsequently inherited to somatic tissues (Wiznerowicz et al. 2007). Although derived from the E3.5 blastocyst, TRIM28 is still essential to endogenous retrovirus (ERV) silencing and imprinting maintenance in ESCs (Rowe et al. 2010; Quenneville et al. 2011). In most differentiated cells, with a few possible exceptions (Fasching et al. 2015), TRIM28 is dispensable for imprint maintenance and ERV silencing. Consequently, shRNA knockdown of Trim28 in ESCs results in marked activation of IAPEz (Fig. 3B; Supplemental Fig. S5A,B). Surprisingly, though, Rbmyla1 is not substantially activated on RNA and is undetectable at the protein level (Fig. 3B; Supplemental Fig. S5A,B). Perhaps derepression alone is insufficient for Rbmyla1 activation due to the lack of specific transcription activators in ESCs, as shown for VL30 ${ }^{\text {Pro }}$ elements in ZFP809 knockout cells (Wolf et al. 2015), or possibly TRIM28 may no longer be required for Rbmy1a1 repression in ESCs, as permanent silencing has been achieved at early embryonic stages. TRIM28 mediates ERV repression through SETDB1 (Schultz et al. 2002; Matsui et al. 2010; Rowe et al. 2010), which, in contrast to TRIM28, is required for the continuous silencing of ERVs in somatic tissues (Wolf et al. 2015). In line with and in contrast to the Trim28 knockdown, the Setdb1 knockdown results in a very robust activation of Rbmy1a1 in ESCs (Fig. 3B; Supplemental Fig. S5C,D). Culturing ESCs under $2 \mathrm{i}$ conditions to promote a naïve state did not alter these outcomes (Supplemental Fig. S5E). We thus conclude that Rbmy1a1 can be activated in ESCs, yet TRIM28-mediated repression is restricted to the early embryo.

To test when TRIM28 is required for Rbmy1a1 repression, we examined Rbmy1a1 expression in blastocysts lacking maternal, zygotic, or maternal and zygotic Trim28 (Fig. 3C; Supplemental Fig. S6). Again, Trim $28^{\text {mat } \Delta /+}$ males displayed reproducible yet variable Rbmy1a1 activation. Control and, importantly, "zygotic" heterozygous embryos never expressed Rbmy1a1, the latter allowing exclusion of haploinsufficiency effects. Crucially, zygotic-null embryos did not activate Rbmy1a1 either. The absence of maternal and zygotic Trim28 caused variable Rbmy1a1 activation comparable with Trim $28^{\text {mat } \Delta /+}$ embryos. Therefore, maternal Trim 28 alone is required and sufficient for the effective silencing of Rbmy1a1 in the preimplantation embryo.

Finally, to test whether Rbmyla1 expression could cause the male-specific phenotype, we expressed wildtype or mutated Rbmy1a1 in cells and embryos (Fig. 4). Rbmy1a1 expression significantly impairs colony formation and cell viability, whereas expression of mutated Rbmy1a1 (Supplemental Fig. S7) does not (Fig. 4B-D). Next, we injected wild-type zygotes with Rbmy1a1 RNA variants and monitored development in vitro. Successful expression of RBMY1A1 was shown by immunofluorescence (Fig. 4E). Expression of a frameshift or in-frame deletion mutant mRNA (Supplemental Fig. S7) did not interfere with preimplantation development. Eighty percent $(n=48$ out of 60$)$ and $86 \%(n=44$ out of 51$)$ of embryos developed to expanded blastocysts, respectively (Fig. 4F,G). Conversely, wild-type Rbmy1a1 mRNA caused developmental arrest in most embryos $(91 \% ; n=126$ out of 138) mainly around the eight-cell to morula stage, underlining the harmful effects of RBMY1A1 outside of its endogenous (testis-specific) expression domain.

Just as for genomic imprints (Bartolomei 2009; Ferguson-Smith 2011; Messerschmidt et al. 2012; Tomizawa 

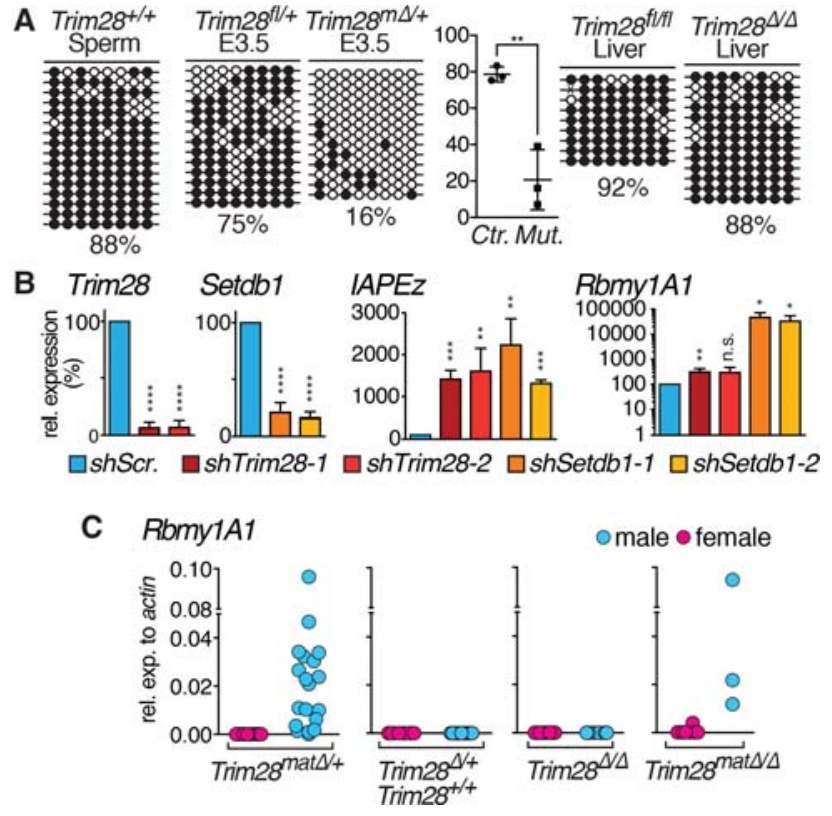

Figure 3. TRIM28-mediated Rbmy1a1 repression is embryo-specific and requires maternal Trim 28 contribution. (A) Rbmy1a1 promoter methylation analysis in wild-type sperm, control and Trim28 $28^{\text {mat } \Delta /+}$ E3.5 blastocysts, and control and Trim28 knockout livers. For blastocysts, whole litters were pooled for analysis. Results for one representative litter are shown. Rbmy1a1 promoter methylation differs significantly between control and Trim2 $28^{\text {mat } \Delta /+}$ E3.5 blastocysts (three of each. (B) Trim28, Setdb1, IAPEz, and Rbmy1a1 (note the logarithmic scale) expression analysis after shRNA knockdown of Trim28 or Setdb1 using two independent shRNAs, respectively, in ESCs. (C) Rbmy1a1 expression analyzed in male and female E3.5 blastocysts with or without maternal and/or zygotic Trim28 contribution.

and Sasaki 2012; Lorthongpanich et al. 2013), we propose that maintenance of epigenetic states at yet undefined genomic loci evading epigenetic reprogramming are vital and that defects within impair development or cause disease. Rbmy1a1 is such a novel gene, and the absence of maternal TRIM28 results in erroneous demethylation, and activation of Rbmy1a1 causes developmental arrest. Because Rbmy1a1 is Y-linked, the phenotype is male-specific. Remarkably, like paternal imprints (Messerschmidt et al. 2012; Lorthongpanich et al. 2013), the Rbmy1a1 locus is exposed only to the TRIM28-null environment post-fertilization; the defect arises within the embryo. The precise timing of TRIM28 requirement is evident, as only maternal but not zygotic TRIM28 is required for Rbmy1a1 repression. Rbmy1a1 detected in two-cell stage mutants also supports the notion of a very early requirement of TRIM28. Furthermore, unlike other embryonic TRIM28 functions identified to date (i.e., imprint maintenance and ERV repression), Rbmy1a1 derepression could not be recapitulated in ESCs. Finally, Rbmy1a1 promoter hypomethylation is detectable as early as the two-cell stage (Supplemental Fig. S8A). Hence, a specialized requirement of TRIM28 at the Rbmy1a1 locus, possibly counteracting active DNA demethylation (for review, see Messerschmidt et al. 2014) or perhaps targeting DNMTs for remethylation in the zygote (Amouroux et al. 2016), is plausible.

Retrotransposons, in particular IAPs, are silenced by TRIM28 in ESCs and embryos (Rowe et al. 2010). As sev- eral IAPEzs are found near Rbmy1a1, their derepression may indirectly induce neighboring Rbmy1a1 gene copies. However, while IAPEzs are activated in Trim28 knockdown ESCs and zygotic-null Trim28 blastocysts (Fig. 3; Rowe et al. 2010), neither shows comparable activation of Rbmy1a1. In line with this, we could not identify IAPEz/Rbmy1a1 chimeric transcripts in Trim $28^{\text {mat } \Delta /+}$ embryos and did not observe DNA methylation changes in an intronic IAPEz insertion at one Rbmyla1 gene copy (Supplemental Fig. S8B,C; data not shown). Thus, while we cannot exclude that minor indirect activation of Rbmy1a1 is driven by neighboring ERVs (see weak activation in Trim28 shRNA knockdown ESCs), the overall derepression of Rbmy1a1 is likely IAPEz-independent if not fully ERV-independent.

RBMY1A1 expression in usually restricted to the testis, where it is reported to modulate splicing events (Mahadevaiah et al. 1998; Zeng et al. 2008; Liu et al. 2009). Possibly, activation of Rbmy1a1 drives the accumulation of testis-specific or aberrant splice variants and protein products in in the embryo, ultimately triggering apoptosis. Aptly, injection of Rbmy1a1 mRNA does not immediately impact on embryo viability, suggesting a possible disturbance in mRNA processing after embryonic gene activation. RT-PCR and cloning of Rbmyla1 from Trim2 $8^{\text {mat } \Delta /+}$ embryos unveiled abnormal splice variants ridden with skipped exons and alternative splice donor/ acceptor sites, causing large deletions and out-of-frame truncations (Supplemental Fig. S9). Targeting its own mRNA in testis for alternative splicing (Zeng et al. 2008, 2011), these severe defects may be attributed to expression of RBMY1A1 in the unnatural embryonic environment. Indeed, analysis of RNA sequencing (RNA-seq) reads revealed a number of gene products with exon-skipping events, which was validated for three genes (Scamp4, Mettl6, and Mybl2) by RT-PCR in individual embryos of both sexes and genotypes (Supplemental Table 4; Supplemental Fig. S10).

All in all, our work reveals a new and unexpected genomic region critically needing protection from DNA demethylation during embryonic epigenetic reprogramming. While, in this scenario, hypomethylation triggers immediate gene activation and embryonic lethality, other yet unidentified Trim28-dependent regions may impact on later development or cause disease/syndromes in the adult. Our findings thus confirm the initial hypothesis and pave the way for future genome-wide studies to expose novel targets beyond the known imprinted regions and Rbmy1a1.

\section{Materials and Methods}

\section{Mice}

Trim28 f/f and Zp3-cre mice were described previously (Messerschmidt et al. 2012). Trim28f/f mice were crossed with $\mathrm{Alb}-\mathrm{Cre} \mathrm{C}^{2}$ to generate Trim28 ${ }^{\text {LiverKO }}$ mice (Postic et al. 1999). Tg(CAG-EGFP)D4Nagy mice (Hadjantonakis et al. 1998) were obtained from the Jackson laboratory and crossed back to C57BL/6J. All mouse work was performed according to Institutional Animal Care and Use Committee regulations.

\section{Embryo isolation}

Embryos were isolated as described after natural mating or superovulation (Behringer et al. 2013). For superovulation, 5 IU of PMSG and, $48 \mathrm{~h}$ later, 5 IU of HCG were given intraperitoneally before mating. 

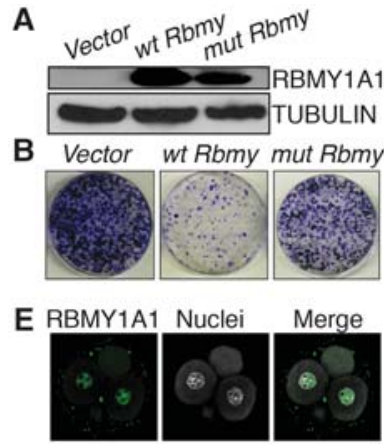

F p-mt Rbmy1a1 wt Rbmy1a1

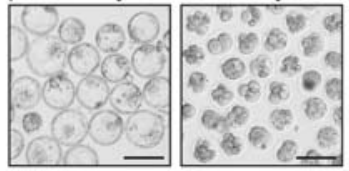

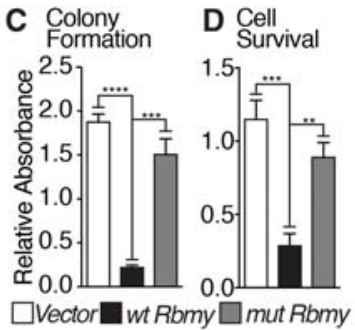

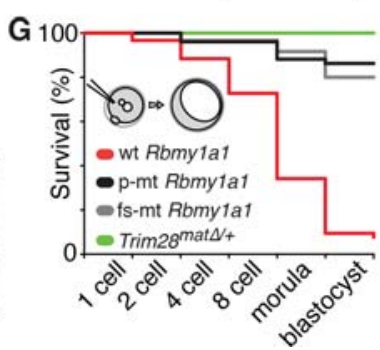

Figure 4. Forced Rbmy1a1 expression hampers cell growth and embryo development. (A) Western blot of cells transduced with vectoronly, wild-type, or mutated Rbmy1a1. (B) Images of colony formation assay using cells described in $A$. $(C)$ Quantification of colony formation assay across three independent experiments. $(D)$ Quantification of MMTS cell survival assay across three independent experiments. (E) RBMY1A1 staining of a four-cell stage embryo injected with wild-type Rbmy1a1 mRNA. (F) Overview of embryos injected with mutant or wild-type Rbmy1a1 after $4 \mathrm{~d}$ of in vitro culture. $(G)$ Quantification of embryo survival throughout in vitro preimplantation development after injection of wild-type Rbmy1a1 mRNA and Rbmy1a1 mRNA variants with a five-amino-acid in-frame deletion or an early frameshift mutation. Untreated Trim $28^{\text {mat } \Delta /+}$ embryos show no preimplantation defects. Bars, $100 \mu \mathrm{m}$.

\section{Embryo mRNA injection}

In vitro transcribed mRNA was diluted in water, and $\sim 1 \mathrm{pL}$ was microinjected into the paternal pronucleus following the standard protocol (Behringer et al. 2013). Injected embryos were cultured at $37^{\circ} \mathrm{C}$ and $5 \% \mathrm{CO}_{2}$ in KSOM + AA medium (Millipore).

\section{RNA isolation and $q R T-P C R$ of cells and preimplantation embryos}

RNA from cells was isolated using RNeasy minikit (Qiagen), and $1 \mu \mathrm{g}$ of total RNA was used for reverse transcription. RNA from embryos was isolated using the PicoPure RNA isolation kit (Fisher Scientific) and used for reverse transcription using the High-Capacity cDNA reverse transcription kit (Applied Biosystems) using random hexamer primers. qPCR was performed using Taq-Man Fast Universal PCR master mix (Applied Biosystems) in combination with the universal probe library (Roche) on a CFX384 Touch real-time PCR system (Bio-Rad). Relative gene expression $\left(2^{\Delta \mathrm{Ct}}\right.$ method) was calculated using the housekeeping gene Actin to normalize the target genes. The primers and probes used are listed in Supplemental Table 5.

\section{Single-embryo RNA-seq}

Maternal mutant or control male embryos were selected for RNA isolation, reverse transcription (Smart Seq kit, Clonetech), and library preparation (Nextera XT kit, Illumina).

RNA-seq reads were mapped to the $\mathrm{mm} 9$ mouse genome assembly using STAR version 2.4.2a (https://github.com/alexdobin/STAR; Dobin et al. 2013) with the following parameters: -sjdbOverhang 99 -outSAMstrandField intronMotif -outSAMtype BAM Unsorted -outFilterMultimapN$\max 40$-twopassMode Basic. Aligned reads were quantified for expression using Cuffdiff (version 2.2.1) (Trapnell et al. 2013). Downstream

manipulation of RNA-seq results were done with in-house scripts and CummeRbund version 2.14.0. BigWig files were generated using bamCoverage from the deepTools package (http://deeptools.readthedocs.io/ en/latest; Ramírez et al. 2014). Data are available online under GSE87504.

\section{Immunofluorescence staining of preimplantation embryos}

Preimplantation embryos were stained as described previously (Messerschmidt and Kemler 2010). The antibodies and dilutions used were $\alpha$ TRIM28 (1:100; Abcam), a-OCT4 (1:100; Santa Cruz Biotechnology), $\alpha$ NANOG (1:100) (Messerschmidt and Kemler 2010), a-CDX2 (1:100; BioGenex), a-Caspase 3 (1:100; Cell Signaling), a-H3K27me3 (1:100; Abcam), and $\alpha$-RBMY (1:100; Santa Cruz Biotechnology). Secondary antibodies (Alexa fluor 488 and 594, Invitrogen) were used at 1:250-500 dilution.

\section{Cell culture}

E14 ESCs were cultured in DMEM with 15\% ES-grade FBS (Gibco) and $1000 \mathrm{U} / \mathrm{mL}$ LIF (Millipore) or under standard 2i conditions. NIH3T3 cells were cultured in DMEM with $10 \%$ FBS. For colony formation assay, cells were seeded at a density of $1 \times 10^{3}$ cells per $10-\mathrm{cm}$ plate. Colonies were fixed and stained with $0.02 \%$ Crystal violet. Stained colonies were first photographed and then extracted in $1 \%$ SDS solution and quantified at $570 \mathrm{~nm}$. For the cell viability assay (MTT), cells were seeded in 96-well plates at a density of 3000 cells per well. MTT solution was added to the cells at a final concentration of $0.5 \mathrm{mg} / \mathrm{mL}$, and cells were incubated at $37^{\circ} \mathrm{C}$ before quantification.

\section{shRNA constructs, transfection, and lentivirus generation}

shRNA oligonculeotides (Supplemental Table 5) were cloned into AgeI/ EcoRI sites of pLKO.1-puro vector. Lentivirus was generated by transfection of pLKO.1 shRNA constructs with packaging and envelope plasmids into 293T cells using Lipofectamine 2000 (Life Technologies). After 24$48 \mathrm{~h}$, viral supernatant was harvested, filtered, and used to transduce E14 ESCs (seeded 16-24 h earlier) along with $80 \mu \mathrm{g} / \mathrm{mL}$ polybrene (Sigma-Aldrich). The infected cells were selected with $2 \mu \mathrm{g} / \mathrm{mL}$ puromycin (Sigma-Aldrich) $48 \mathrm{~h}$ after infection.

\section{Protein isolation and Western blot analysis}

Cells were lysed and extracted with RIPA buffer supplemented with protease inhibitors (Roche). Twenty micrograms of total protein was loaded onto a Tris-glycine SDS-polyacrylamide gel, separated, and transferred to nitrocellulose membrane (Bio-Rad). The membrane was blocked and then incubated with primary antibody overnight. The membrane was washed and incubated with HRP-conjugated secondary antibody before detection with chemiluminescent HRP substrate (Millipore) and exposure. The antibodies and dilutions used were $\alpha$-TRIM28 (1:1000; Abcam), a-SetDB1 (1:500; Santa Cruz Biotechnology), $\alpha$ - $\alpha$-tubulin (1:1000-5000; Sigma-Aldrich), and Rbmylal (1:500-1000; Santa Cruz Biotechnology).

\section{Histology}

Deciduomas were isolated at E6.5 and fixed in 4\% PFA, processed for paraffin embedding, and sectioned. Rehydrated sections were stained with Harris hematoxylin (Sigma Aldrich) and counterstained with eosin Y (Sigma-Aldrich). Sections were dehydrated and mounted in DPX (SigmaAldrich).

\section{DNA methylation analysis}

DNA methylation was analyzed by bisulfite conversion, cloning, and sequencing as described before (Messerschmidt et al. 2012). Briefly, DNA from a pooled litter of E3.5 embryos was used for bisulfite conversion according to the manufacturer's protocol (Imprint DNA modification kit, Sigma). For sperm, ESCs, or tissue samples, $1 \mu \mathrm{g}$ of genomic DNA was used for conversion. PCR fragments were cloned and sequenced. Primers are listed in Supplemental Table S5. 


\section{Statistical analysis}

If not otherwise stated, Student's $t$-test was performed. "n.s." indicates not significant $(P>0.05)$, single asterisks indicate $P=0.01-0.05$, double asterisks indicate $P=0.001-0.01$, and triple asterisks indicate $P=0.0001$ 0.001 or $P<0.0001$.

\section{Acknowledgments}

We thank Phillipp Kaldis, Barbara B. Knowles, and Davor Solter for discussions and advice. This work was supported by a National Research Foundation (NRF) Fellowship and a Young Investigator Grant by the Biomedical Research Council of the Agency for Science, Technology, and Research (A*STAR) of Singapore to D.M.M.

\section{References}

Amouroux R, Nashun B, Shirane K, Nakagawa S, Hill PWS, D'Souza Z, Nakayama M, Matsuda M, Turp A, Ndjetehe E, et al. 2016. De novo DNA methylation drives $5 \mathrm{hmC}$ accumulation in mouse zygotes. Nat Cell Biol 18: 225-233.

Bartolomei MS. 2009. Genomic imprinting: employing and avoiding epigenetic processes. Genes Dev 23: 2124-2133.

Behringer R, Gertsenstein M, Nagy KV, Nagy A. 2013. Manipulating the mouse embryo. Cold Spring Harbor Laboratory Press, Cold Spring Harbor, NY.

Branco MR, King M, Perez-Garcia V, Bogutz AB, Caley M, Fineberg E, Lefebvre L, Cook SJ, Dean W, Hemberger M, et al. 2016. Maternal DNA methylation regulates early trophoblast development. Dev Cell 36: 152-163.

Cammas F, Mark M, Dollé P, Dierich A, Chambon P, Losson R. 2000. Mice lacking the transcriptional corepressor TIF $1 \beta$ are defective in early postimplantation development. Development 127: 2955-2963.

Dobin A, Davis CA, Schlesinger F, Drenkow J, Zaleski C, Jha S, Batut P, Chaisson M, Gingeras TR. 2013. STAR: ultrafast universal RNA-seq aligner. Bioinformatics 29: 15-21.

Fasching L, Kapopoulou A, Sachdeva R, Petri R, Jönsson ME, Männe C, Turelli P, Jern P, Cammas F, Trono D, et al. 2015. TRIM28 represses transcription of endogenous retroviruses in neural progenitor cells. Cell Rep 10: 20-28.

Ferguson-Smith AC. 2011. Genomic imprinting: the emergence of an epigenetic paradigm. Nat Rev Genet 12: 565-575.

Hadjantonakis AK, Gertsenstein M, Ikawa M, Okabe M, Nagy A. 1998. Non-invasive sexing of preimplantation stage mammalian embryos. Nat Genet 19: 220-222.

Hirasawa R, Chiba H, Kaneda M, Tajima S, Li E, Jaenisch R, Sasaki H. 2008. Maternal and zygotic Dnmtl are necessary and sufficient for the maintenance of DNA methylation imprints during preimplantation development. Genes Dev 22: 1607-1616.

Liu Y, Bourgeois CF, Pang S, Kudla M, Dreumont N, Kister L, Sun Y-H, Stévenin J, Elliott DJ. 2009. The germ cell nuclear proteins hnRNP G-T and RBMY activate a testis-specific exon. PLoS Genet 5: e1000707.

Lorthongpanich C, Cheow LF, Balu S, Quake SR, Knowles BB, Burkholder WF, Solter D, Messerschmidt DM. 2013. Single-cell DNA-methylation analysis reveals epigenetic chimerism in preimplantation embryos. Science 341: 1110-1112.

Mahadevaiah SK, Odorisio T, Elliott DJ, Rattigan A, Szot M, Laval SH, Washburn LL, McCarrey JR, Cattanach BM, Lovell-Badge R, et al. 1998. Mouse homologues of the human AZF candidate gene RBM are expressed in spermatogonia and spermatids, and map to a Y chromosome deletion interval associated with a high incidence of sperm abnormalities. Hum Mol Genet 7: 715-727.
Matsui T, Leung D, Miyashita H, Maksakova IA, Miyachi H, Kimura H, Tachibana M, Lorincz MC, Shinkai Y. 2010. Proviral silencing in embryonic stem cells requires the histone methyltransferase ESET. Nature 464: 927-931.

Messerschmidt DM, Kemler R. 2010. Nanog is required for primitive endoderm formation through a non-cell autonomous mechanism. Dev Biol 344: 129-137.

Messerschmidt DM, de Vries W, Ito M, Solter D, Ferguson-Smith A, Knowles BB. 2012. Trim28 is required for epigenetic stability during mouse oocyte to embryo transition. Science 335: 1499-1502.

Messerschmidt DM, Knowles BB, Solter D. 2014. DNA methylation dynamics during epigenetic reprogramming in the germline and preimplantation embryos. Genes Dev 28: 812-828.

Postic C, Shiota M, Niswender KD, Jetton TL, Chen Y, Moates JM, Shelton KD, Lindner J, Cherrington AD, Magnuson MA. 1999. Dual roles for glucokinase in glucose homeostasis as determined by liver and pancreatic $\beta$ cell-specific gene knock-outs using Cre recombinase. I Biol Chem 274: 305-315.

Quenneville S, Verde G, Corsinotti A, Kapopoulou A, Jakobsson J, Offner S, Baglivo I, Pedone PV, Grimaldi G, Riccio A, et al. 2011. In embryonic stem cells, ZFP57/KAP1 recognize a methylated hexanucleotide to affect chromatin and DNA methylation of imprinting control regions. Mol Cell 44: 361-372.

Ramírez F, Dündar F, Diehl S, Grüning BA, Manke T. 2014. deepTools: a flexible platform for exploring deep-sequencing data. Nucleic Acids Res 42: W187-W191.

Rowe HM, Jakobsson J, Mesnard D, Rougemont J, Reynard S, Aktas T, Maillard PV, Layard-Liesching H, Verp S, Marquis J, et al. 2010. $\mathrm{KAP} 1$ controls endogenous retroviruses in embryonic stem cells. $\mathrm{Na}$ ture 463: 237-240.

Rowe HM, Friedli M, Offner S, Verp S, Mesnard D, Marquis J, Aktas T, Trono D. 2013. De novo DNA methylation of endogenous retroviruses is shaped by KRAB-ZFPs/KAP1 and ESET. Development 140: 519-529.

Schultz DC, Ayyanathan K, Negorev D, Maul GG, Rauscher FJ. 2002. SETDB1: a novel KAP-1-associated histone H3, lysine 9-specific methyltransferase that contributes to HP1-mediated silencing of euchromatic genes by KRAB zinc-finger proteins. Genes Dev 16: 919-932.

Soh YQS, Alföldi J, Pyntikova T, Brown LG, Graves T, Minx PJ, Fulton RS, Kremitzki C, Koutseva N, Mueller JL, et al. 2014. Sequencing the mouse $\mathrm{Y}$ chromosome reveals convergent gene acquisition and amplification on both sex chromosomes. Cell 159: 800-813.

Tomizawa S-I, Sasaki H. 2012. Genomic imprinting and its relevance to congenital disease, infertility, molar pregnancy and induced pluripotent stem cell. J Hum Genet 57: 84-91.

Trapnell C, Hendrickson DG, Sauvageau M, Goff L, Rinn JL, Pachter L. 2013. Differential analysis of gene regulation at transcript resolution with RNA-seq. Nat Biotechnol 31: 46-53.

Wiznerowicz M, Jakobsson J, Szulc J, Liao S, Quazzola A, Beermann F, Aebischer P, Trono D. 2007. The Kruppel-associated box repressor domain can trigger de novo promoter methylation during mouse early embryogenesis. J Biol Chem 282: 34535-34541.

Wolf G, Yang P, Füchtbauer AC, Füchtbauer E-M, Silva AM, Park C, Wu W, Nielsen AL, Pedersen FS, Macfarlan TS. 2015. The KRAB zinc finger protein ZFP809 is required to initiate epigenetic silencing of endogenous retroviruses. Genes Dev 29: 538-554.

Zeng M, Sun H, Chen S, Wang X, Yang Y, Liu Y, Tao D, Yang Z, Zhang S, Ma Y. 2008. Identification of target messenger RNA substrates for mouse RBMY. Mol Hum Reprod 14: 331-336.

Zeng M, Liang S, Zhao S, Liu Y, Sun H, Zhang S, Ma Y. 2011. Identifying mRNAs bound by human RBMY protein in the testis. J Reprod Dev 57: 107-112. 


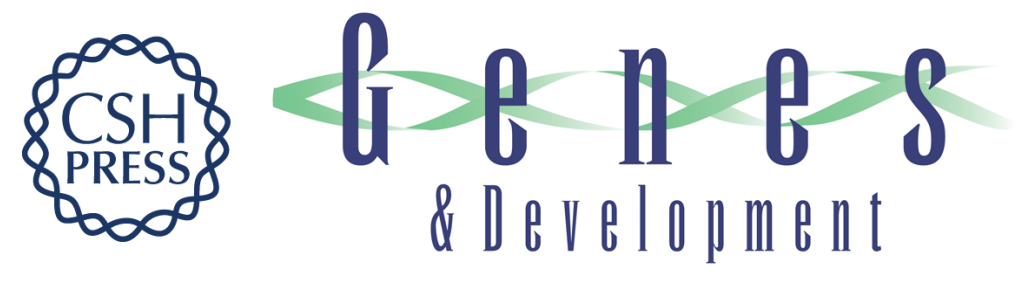

\section{Loss of maternal Trim28 causes male-predominant early embryonic lethality}

Abhishek Sampath Kumar, Michelle K.Y. Seah, Ka Yi Ling, et al.

Genes Dev. 2017, 31: originally published online January 23, 2017

Access the most recent version at doi:10.1101/gad.291195.116

\section{Supplemental http://genesdev.cshlp.org/content/suppl/2017/01/23/gad.291195.116.DC1 Material}

References This article cites 29 articles, 11 of which can be accessed free at: http://genesdev.cshlp.org/content/31/1/12.full.html\#ref-list-1

Creative This article, published in Genes \& Development, is available under a Creative Commons Commons License (Attribution 4.0 International), as described at License http://creativecommons.org/licenses/by/4.0/.

Email Alerting Receive free email alerts when new articles cite this article - sign up in the box at the top Service right corner of the article or click here.

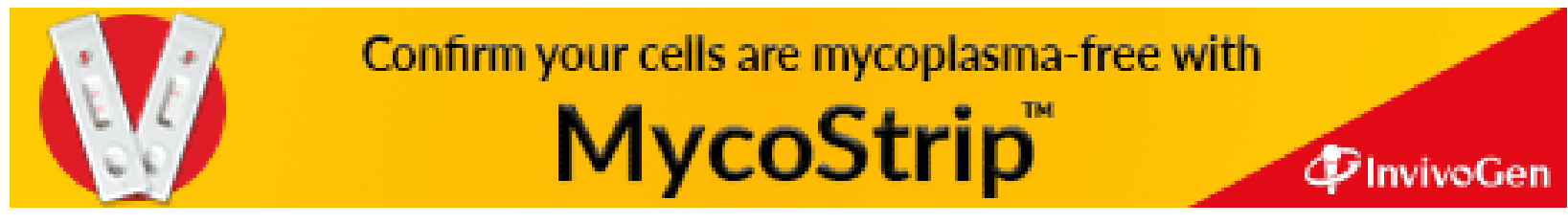

